\title{
Repercusiones neuropsicológicas del consumo de bazuco: una revisión de la literatura ${ }^{9}$
}

\author{
Héctor Fabio Rojas Espitia \\ Psicólogo \\ Egresado Fundación Universitaria de Ciencias de la Salud - FUCS \\ Correo electrónico: hfrojas@fucsalud.edu.co \\ Martha Lucía Triviño Luengas \\ Psicóloga \\ Egresado Fundación Universitaria de Ciencias de la Salud - FUCS \\ Correo electrónico: mltrivino@fucsalud.edu.co
}

\section{Ana Lucía Guzmán Durán}

Neuropsicóloga Investigadora Fundación Universitaria de Ciencias de la Salud - FUCS

Correo electrónico: alguzman@fucsalud.edu.co

\section{Hernando Andrés Olaya Acosta}

Especialista en Toxicología Clínica Docente Fundación Universitaria de Ciencias de la Salud - FUCS

Correo electrónico: holaya@fucsalud.edu.co
Recibido: 16/01/2018

Evaluado: $17 / 07 / 2018$

Aceptado: $10 / 12 / 2018$

\section{Resumen}

Objetivo: realizar una revisón de la literatura sobre las consecuencias neuropsicológicas y la alteración de las estructuras cerebrales en consumidores de bazuco. Método: se realizó una búsqueda en las bases de datos ScienceDirect, PubMed, EBSCO, PsycINF0, Ovid, Redalyc, Dialnet y Google Schoolar; utilizando como descriptores: basuco, bazuco, cocaína, cognición, atención, memoria, funciones ejecutivas y disfunción cognitiva, tanto en español como en inglés. Resultados: se incluyeron 30 artículos publicados entre 1994 y 2015 en los que se encontró hipoactividad de la corteza prefrontal, déficits neurocognitivos en el componente atencional, mnésico y ejecutivo, así como un decremento en habilidades visoespaciales, psicomotrices y verbales. Además de ello, se presentan cambios comportamentales como agresividad, impulsividad y menor inhibición de respuestas. Se concluye la necesidad de realizar investigaciones con diseños longitudinales que permitan esclarecer las alteraciones neuropsicológicas a largo plazo, además de delimitar criterios de inclusión en donde los participantes no sean policonsumidores.

Palabras clave Atención, bazuco, cognición, función ejecutiva, memoria y pasta base de cocaína.

9 Para citar este artículo: Rojas, H.F., Triviño, M.L., Guzmán, A.L. \& Olaya, A. (2019). Repercusiones neuropsicológicas del consumo de bazuco: una revisión de la literatura. Informes Psicológicos, 19(2), pp. 125-142 http://dx.doi. org/10.18566/infpsic.v19n2a09 


\title{
Neuropsychological repercussions of bazuco consumption: a literature review
}

\begin{abstract}
Objective: to carry out a review of the literature on the neuropsychological consequences and the alteration of brain structures in bazuco consumers. Method: a search was made in the databases ScienceDirect, PubMed, EBSCO, PsycINF0, Ovid, Redalyc, Dialnet and Google Scholar, using as descriptors: basuco, bazuco, cocaine, cognition, attention, memory, executive functions and cognitive dysfunction, both in Spanish and in English. Results: Thirty articles published between 1994 and 2015 were included in which hypoactivity of the prefrontal cortex was found, as well as neurocognitive deficits in the attentional, mnemonic and executive component, and a decrease in visuospatial, psychomotor and verbal abilities. In addition, behavioral changes such as aggressiveness, impulsivity and a lesser inhibition of responses are presented. We conclude the need to carry out investigations with longitudinal designs that help to clarify the long-term neuropsychological alterations, as well as to define inclusion criteria where the participants are not polyconsumers.
\end{abstract}

\section{Repercussões neuropsicológicas do consumo de bazuco: uma revisão da literatura}

\section{Resumo}

Objetivo: realizar uma revisão da literatura sobre as consequências neuropsicológicas e a alteração de estruturas cerebrais em consumidores de bazuco. Realizou-se um levantamento de informação nas bases de dados ScienceDirect, PubMed, EBSCO, PsycINFO, Ovid, Redalyc, Dialnet y Google Schoolar; usando como descritores: basuco, bazuco, cocaína, cognição, atenção, memória, funções executivas e disfunção cognitiva, tanto em espanhol quanto em inglês. Resultados: incluíram-se 30 artigos publicados entre 1994 e 2015 nos quais se achou hipoatividade do córtex pré-frontal, déficit neurocognitivo, déficits neurocognitivos nos componentes atencionais, mnemônicos e executivos, bem como diminuição das habilidades visuoespaciais, psicomotoras e verbais. Além disso, mudanças comportamentais como agressividade, impulsividade e menor inibição das respostas são apresentadas. Conclui-se a necessidade de realizar investigações com delineamentos longitudinais que esclareçam as alterações neuropsicológicas a longo prazo, bem como definam critérios de inclusão em que os participantes não sejam poli consumidores.

Palavras chave Atenção, bazuco, cognição, função executiva, memória, pasta base de cocaína. 


\section{ntroducción}

El bazuco es una sustancia psicoactiva que recibe diferentes denominaciones de acuerdo al lugar en el que se fabrique, por ejemplo, en Argentina y en Uruguay se conoce como "paco", en Bolivia como "pitillo", y como "pasta base de cocaína" en Chile (Oficina de las Naciones Unidas contra la Droga y el Delito, 2013). Su consumo en Ámerica del sur se encuentra asociado a las dificultades económicas de la década de los años 80, llevando a que los sectores marginados y en condición de vulnerabilidad incrementaran la producción de clorhidrato de cocaína. Esta situación condujo a una mayor facilidad en el acceso y adicción a esta sustancia, derivando en una problemática de salud pública en los países Latinoamericanos (Meikle et al., 2009; Organización de los Estados Americanos y Comisión Interamericana para el Control del Abuso de Drogas, 2014). En la actualidad, el consumo de bazuco se ha presentado en personas pertenecientes a todas las clases sociales, siendo éste un factor que acentúa la importancia de generar estrategias que permitan mitigar las alteraciones que repercuten en la salud de los consumidores (UNODC, 2013; Uribe, 2009).

En Colombia, se estimó que la tasa de utilización del bazuco para el año 2008 fue de $1.1 \%$ en la población general, índice que podría ser superior al incluir segmentos vulnerables de la población (Scoppetta, 2010). Alrededor de 49.000 personas reportaron consumirlo alguna vez en su vida, indicando una edad media de inicio de consumo de 19 años para ambos sexos (Gobierno Nacional de la República de Colombia, 2014). No obstante, se observa una prevalencia superior en los hombres con un aproximado de 45.000 casos y un patrón de consumo de abuso o dependencia en el 70\% de las personas entre 25 y 34 años (Gobierno Nacional de la República de Colombia, 2014).

En cuanto a su composición, Sabogal y Urrego (2012) describen que es un polvo blanco-amarillento mate, volátil y sublimable, consumido de forma similar a un cigarrillo. Se obtiene como un subproducto de la técnica utilizada para extraer base de coca, empleando solventes que disminuyen la cantidad de cocaína y aumentan la presencia de otras sustancias como el kerosene y el ácido sulfúrico. Estos componentes son los responsables de ocasionar efectos anestésicos, estimulación del sistema nervioso central (SNC) e inhibición de la recaptación de catecolaminas, en un tiempo que oscila de uno a dos minutos tras la inhalación (Benowitz, 2004; Casale \& Klein, 1993; Córdoba \& Toledo, 2001).

El consumo continuo de la sustancia se víncula con agitación psicomotriz, anorexia, pérdida de peso, miocardiopatías, insuficiencia renal, deterioro mental y alucinaciones durante la fase de intoxicación aguda (Damin \& Grau, 2015). Por el contrario, las sensaciones desagradabes a nivel biológico, psicológico y comportamental experiementadas en la fase de abstinencia, conducen a la ejecución de conductas de búsqueda y consumo que refuerzan la adicción por la activación del sistema de recompensa (López \& Becoña, 2006). Refuerzo que es atribuido al aumento de la actividad 
dopaminérgica en el sistema mesocorticolímbico que incluye el área tegmental ventral (ATV) y el núcleo accumbens (ACC) (Fernández, 2006).

El estudio de la repercursiones neuropsicológicas asociadas con el consumo de bazuco continua siendo un desafío para el desarrollo de investigaciones que permitan identificar el perfil cognitivo, considerando que los primeros hallazgos han reportado disfunciones atencionales, mnésicas y del funcionamiento ejecutivo (Rosselli, Rico, Sandoval, Sierra \& Uribe, 1994). Estas disfunciones han sido explicadas por Ferrando et al. (2009) al hallar hipoactividad en la perfusión cerebral de los circuitos prefrontales-subcorticales, estructuras límbicas y corteza de asociación en consumidores de pasta base de cocaína. A pesar de esto, los estudios preliminares no permiten esclarecer los principales déficits en el rendimiento cognitivo al no existir un acuerdo con respecto a las alteraciones en los subtipos de atención y memoria. Por esta razón, los resultados obtenidos mediante diseños metodológicos transversales y longitudinales son contradictorios, lo que dificulta la generalización de sus hallazgos al utilizar diferentes instrumentos de evaluación neuropsicológica y de neuroimagen que no se ajustan a toda la población.

En este contexto se pone de manifiesto la importancia de establecer con mayor precisión el desempeño cognitivo de esta población, con el fin de desarrollar programas de rehabilitación neuropsicológica que disminuyan la tasa de recaída. De ahí que la mejoría en el funcionamiento cognitivo implica para los sujetos un mejor control inhibitorio, automonitoreo y planeación de la conducta, llevando consigo una reducción de las conductas de búsqueda y consumo de la sustancia (Samoel-Rodrigues et al., 2017).

Dada la problemática expuesta, el objetivo fue realizar una revisón de la literatura sobre las consecuencias neuropsicológicas y la alteración de las estructuras cerebrales en consumidores de bazuco.

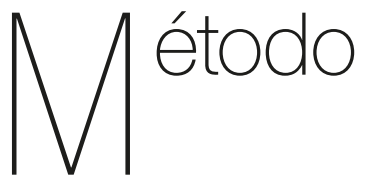

Para el desarrollo de la presente revisión de la literatura se llevó a cabo una búsqueda en las bases de datos ScienceDirect, PubMed, EBSCO, PsycINFO, Ovid, Redalyc, Dialnet y Google Schoolar; utilizando la combinación de los descriptores: basuco, bazuco, cocaína, cognición, atención, memoria, funciones ejecutivas y disfunción cognitiva, tanto en español como en inglés. Fueron seleccionados los artículos publicados entre el 2005 al 2017, sin embargo, se incluyeron estudios publicados antes de la fecha establecida dada la pertinencia de sus hallazgos. Asimismo, se tuvieron en cuenta artículos en los que se presentan las alteraciones neuropsicológicas a la exposición de solventes como benzeno, tolueno, xileno, plomo y permanganato de potasio al ser componentes del bazuco. Se realizó la lectura de los artículos que mencionaran en su título o resumen uno de los descriptores mencionados, para posteriormente filtrar aquellos que permitieran el cumplimiento del objetivo de la revisión. 


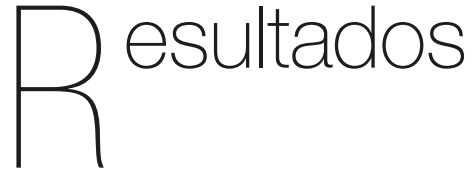

\section{Composición y vía de administración}

El bazuco se caracteriza por ser una sustancia untuosa de consistencia pastosa, color blanco-amarillento, olor penetrante, sabor astringente y poco soluble en agua, como resultado de los tres mecanismos de obtención más comunes, siendo ellos: 1) extracción de cristales de cocaína; 2) refinación de la cocaína junto con adición de agua y amoníaco y 3) utilización de los residuos obtenidos tras el proceso de refinación del clorhidrato de cocaína, para finalmente ser consumido (Castaño, 2000; Motta, 1984). En síntesis, es un producto intermedio en la fabricación de clorhidrato de cocaína derivado de las hojas de coca, las cuales son disueltas en agua y posteriormente tratadas en una solución con ácido sulfúrico, al que se agrega un agente oxidante como el permanganato de potasio para finalizar con la adición de sustancias como petróleo, polvo de ladrillo, entre otros (Dreisbach, Robertson \& William, 1999).

En cuanto a la composición química del bazuco, Sabogal y Urrego (2012) describen la presencia de cocaína, cafeína y fenacitina; no obstante, la gran variedad de sustancias utilizadas en su fabricación no permite generalizar su composición dadas las variaciones en cada país. Un ejemplo de lo anterior es la descripción realizada por Sánchez (2006), citando al Hospital Universitario San Vicente de Paúl, en la que se menciona que:
Los componentes del bazuco son: cocaína en $14 \%$, otros alcaloides de la coca, colorantes y restos vegetales, sustancias saponíferas y alcalinas, trazas de ácido sulfúrico, etanol, metanol, y restos de los sobrantes utilizados en el proceso de obtención como gasolina, kerosene, acetona, éter, cloroformo, etc. Además de los adulterantes que le agregan como azúcar, bicarbonato, harina de trigo, maicena, polvo de ladrillo, etc. (p. 58).

Por último, y dadas las características de composición del bazuco, se ha encontrado que éste tiene un punto de fusión bajo que permite su volatilización y fácil inhalación cuando es fumado, a lo que se suma su baja solubilidad en las membranas mucosas de la nariz o la sangre llevando a que su vía de administración sea la fabricación de cigarrillos, generalmente combinados con tabaco y marihuana (Observatorio Colombiano de Drogas, 2016).

\section{Características clínicas}

\section{Fases de la intoxicación}

A lo largo de estas fases se pueden encontrar síntomas ambigüos que inician con periodos de bienestar, lucidez, energía motora, aumento de la capacidad sexual y, por otra parte, ansiedad, paranoia e inquietud; en casos de consumo crónico son frecuentes los temblores, convulsiones y aumento de temperatura corporal por la activación del sistema nervioso simpático, lo que puede ocasionar taquicardia, hipertensión e infarto al 
miocardio (Organización de los Estados Americanos, Comisión Interamericana para el Control del Abuso de Drogas, Organización Panamericana de la Salud y Organización Mundial de la Salud, 2005). No obstante, cuando los efectos de la sustancia activa del bazuco cesan, los sujetos experimentan disforia, cansancio, irritabilidad y depresión, síntomas que derivan en conductas de búsqueda (O'Brien, 2001). Echeverry, Moya, Zapata y Martínez (2004) han documentado las fases acontecidas tras la inhalación del bazuco en los sujetos:

\section{Fase de euforia}

Su aparición se da a los pocos segundos del consumo, se presenta una sensación de bienestar, excitación placentera, hipersexualidad, hiperactividad, desaparición de la fatiga, disminución del apetito y la necesidad de dormir (Echeverry et al., 2004). Otros autores postulan que en dicha fase hay una disminución en la capacidad de inhibición, aceleración psicomotriz, sobrevaloración de las capacidades, hipervigilancia y distorsión cognitiva (Méndez \& Murillo, 2013).

\section{Fase de disforia}

Posterior al paso por la etapa de euforia, se da la aparición de síntomas disfóricos asociados con sentimientos de angustia que ocasionan gran malestar y llevan a un consumo repetitivo, con el fin de disminuir la intensidad de los síntomas antes descritos; motivo por el que se genera una predisposición en el consumidor para la aparición de alteraciones físicas como infarto miocárdico, hemorragia cerebral, pérdida de peso, agitación psicomotriz, insomnio y taquicardia por el agotamiento de la sustancia al interior del organismo (Sánchez \& Belistri, 2007).

\section{Fase de alucinosis}

Se da tras el consumo de altas dosis y desencadena alucinaciones visuales, táctiles, auditivas y olfatorias, siendo común la percepción de objetos diminutos que pueden producir pánico y conductas agresivas que preceden los intentos de huida en los que se reduce el deseo sexual (Echeverry et al., 2004; Ortega, 2003). Los mecanismos de acción neurobiológica son los responsables de los efectos antes mencionados por el exceso de serotonina en la hendidura sináptica (Gainza et al., 2003).

\section{Fase de psicosis}

Se presentan síndromes confusionales y afectivos, paranoia y agresividad intensa que puede llevar a riesgos para la integridad personal y de terceros (Manschreck et al., 1988). Adicionalmente, el episodio psicótico es una de las complicaciones psicopatológicas más frecuentes asociada al consumo agudo y, por ello, es de corta duración; en algunas ocasiones aparecen con él síntomas depresivos, angustia por el deseo de consumo, hipervigilancia, ideas delirantes paranoides de ser atrapado por la ley o ser víctima de un robo de su dosis (Roncero, Ramos, Collazos \& Casas, 2001).

\section{Mecanismos de acción neurobiológica}

A partir de la vía de administración del bazuco, y de acuerdo con Lizasoain, Moro y Lorenzo (2000), la biodisponibilidad de 
la sustancia se encuentra en una tasa que varía del 10 al 20\%, resaltando que se obtiene una distribución del $70 \%$ en todo el organismo en un periodo de 8 a 10 segundos tras la inhalación. A medida que la sustancia avanza hacia el cerebro a través del plasma sanguíneo, se encuentra con la barrera hematoencefálica, estructura que permite conservar la homeostasis cerebral evitando el ingreso de agentes patógenos y sustancias tóxicas al encéfalo; sin embargo, la cocaína y sus derivados modifican la densidad neuronal al atravesar esta estructura (Urigüen \& Callado, 2010).

De manera simultánea, esta sustancia es transformada al interior del organismo en dos metabolitos inactivos conocidos como: ecgonina y benzoilecgonina, responsables de potenciar la acción de las catecolaminas: dopamina, adrenalina, noradrenalina y serotonina (Garro, 2011). Otros estudios clínicos han identificado que la pirolisis de la cocaína genera un metabolito con mayor capacidad neurotóxica, ocasionando una aceleración en la apoptosis cerebral en el consumo de base libre de cocaína, conocido como Anhydroecgonine Methyl Ester (AEME) al disminuir el metabolismo mitocondrial y la viabilidad neuronal (Areal et al., 2015; García et al., 2012).

El sistema dopaminérgico se ve afectado por la pirolisis de la cocaína al producir el metobolito AEME e impedir la eliminación de la dopamina resultante de la sinapsis, debido al bloqueo de los receptores de la neurona presináptica, por lo que se incrementa la captación en los receptores de la neurona postsináptica; esto se relaciona con el sistema de recompensa y los efectos de euforia inicial que se han reportado en las descripciones clínicas
(García et al., 2012; National Institute of Drug Abuse, 2010). Estos cambios surgen tras la unión del principio activo del bazuco al transportador de recaptación de aminas dado que esta sustancia actua como un bloqueador de los transportadores de monoaminas (Fernández, 2006; Organización de los Estados Americanos, Comisión Interamericana para el Control del Abuso de Drogas, Organización Panamericana de la Salud y Organización Mundial de la Salud, 2005). Por consiguiente, se incrementa la síntesis de dopamina que, a su vez, aumentará la concentración en la hendidura sináptica y los sistemas mesocorticolímbicos, asociados con el sistema de recompensa, gratificación y conductas compulsivas de autoadministración; pero cuando existe un consumo crónico, es posible observar la reducción de la neurotransmisión dopaminérgica que explicaría los síntomas de anhedonia (Souza \& Cruz, 2008).

Respecto al sistema adrenérgico, se ha observado que la ecgonina y la benzoilecgonina impiden la recaptación por el bloqueo del sistema de transporte en membrana, al incrementar la disponibilidad en la hendidura sináptica de adrenalina y noradrenalina (Téllez \& Cote, 2005). Esta disponibilidad lleva consigo una elevación de la tiroxina hidroxilasa, predisponiendo al individuo para el desarrollo de taquicardia e hipertensión por la activación del sistema nervioso autónomo que regula el sistema de alerta y vigilia (Cooper, 1982; Martínez, Aguilar \& Rosales, 2006).

La serotonina es otro de los neurotransmisores afectados tras el consumo, puesto que se produce un bloqueo de los transportadores activos llevando a la acumulación de esta sustancia en la 
hendidura sináptica y, con ello, el aumento en la recaptación de los receptores postsinápticos; lo que explica las propiedades euforizantes de la sustancia, los cambios en el apetito, las alucinaciones y las distorsiones perceptivas (Ambrosio, 2008; Callado, 2001).

\section{Estructuras cerebrales implicadas}

El sistema de recompensa es el encargado de regular los efectos reforzantes, tras la estimulación química del sistema mesocorticolímbico por parte de los sistemas de neurotransmisión dopaminérgicos involucrados en el consumo, por la activación del área tegmental ventral (ATV), el pallidum ventral, y el estriado ventral compuesto por los núcleos caudado, putamen y accumbens, así como la hipoactividad de la corteza frontal (Smith \& Dworkin, 2000).

Los estudios demuestran que el estriado ventral y dorsal presentan cambios morfológicos al ser los responsables de recibir proyecciones glutamatérgicas y dopaminérgicas durante el consumo de bazuco, en ellos se evidencia pérdida de volumen y transformación tisular, resultando en disfunciones en el procesamiento cognitivo de la información y la integración sensoriomotriz (Garza-Villarreal et al., 2017; Schuch-Goi et al., 2017).

Otros autores como Schuch-Goi et al. (2017) han señalado la mediación de estructuras como el núcleo accumbens en la recepción de información proveniente del hipocampo, la amígdala y el lóbulo frontal al considerarse estructuras claves para el control de comportamientos de recompensa vinculados con los procesos de adicción por la recurrencia al consumo. Los altos niveles de dopamina en el núcleo accumbens, generan alteraciones en los sistemas motivacionales y de recompensa, relacionados con conductas motoras evidenciadas en la fase de euforia (Pascale, Negrin \& Laborde, 2010).

La amígdala y el hipocampo son los responsables de los procesos de aprendizaje y memoria al asociarse con una experiencia de alta carga emocional, lo que contribuye a la adquisición, consolidación y manifestación de la conducta implicada en el consumo de drogas, además de presentarse una disminución en su volumen por el daño en los tejidos de estas estructuras (Lima, 2007; Lipaus et al., 2018; Pereiro, 2011).

En resumen, las estructuras involucradas en el sistema de recompensa están reguladas por los circuitos gabérgicos y glutamatérgicos conectados con el sistema dopaminérgico quienes determinan la disfunción de estos circuitos por el incremento del principio activo del bazuco, provocando así que las respuestas de las diferentes estructuras cerebrales se relacionen con los comportamientos de búsqueda que llevan a un mayor consumo de la droga ante las dificultades en el control de impulsos (Delgado, 2011; Volkow, Wang, Fowler, Tomasi \& Telang, 2010).

La exposición al bazuco genera la pérdida del control cognitivo y comportamental, al disminuir la capacidad de automonitoreo, selección de objetivos, toma de decisiones y autocontrol, con el consecuente aumento de las conductas de búsqueda y consumo derivadas de la 
hipoactivación así como una reducción de la corteza prefrontal al observarse daños en la sustancia gris (Batista, Klauss, Fregni, Nitsche, \& Nakamura, 2015; Conti \& Nakamura-Palacios, 2014; Delgado, 2011; Fein, Di Sclafani \& Meyerhoff, 2002).

Otros estudios como el presentado por Bolla et al. (2004), confirman que los consumidores de bazuco presentan menor activación en las regiones del cíngulo anterior izquierdo y la corteza prefrontal lateral derecha; encargadas de la supervisión de errores, resolución de problemas, inhibición de respuestas, supervisión del rendimiento e implementación del control. Por lo que explica que las funciones anteriormente descritas perjudican la habilidad individual de supervisar e inhibir comportamientos inapropiados como el de la búsqueda de droga; no obstante, se encontró una mejor activación del cíngulo derecho en los sujetos adictos que participaron en el estudio.

\section{Perfil cognitivo}

El consumo de bazuco se ha asociado con la existencia de disfunciones cognitivas mediadas por la frecuencia y el tiempo de consumo, sin embargo, los estudios disponibles para esta sustancia aún son escasos, por lo que gran parte de las alteraciones identificadas corresponden al principio activo del bazuco.

Los estudios desarrollados con instrumentos neuropsicológicos han reportado que, tras un consumo crónico del principio activo del bazuco, se presentan importantes alteraciones en funciones cognitivas como la memoria, atención, habilidades visoespaciales, decremento en la velocidad psicomotora y funciones ejecutivas, cuadros delirantes, desinterés laboral y/o académico y comienzo o agravamiento de conductas antisociales (Bolla, Rothman \& Cadet, 1999; Sánchez \& Belistri, 2007). Madoz y Ochoa (2012) confirman los hallazgos reportados a través de la aplicación de cuatro pruebas neuropsicológicas en una muestra de 27 consumidores en los que se encuentran rendimientos inferiores al grupo control y a los baremos de referencia en: atención, memoria de trabajo, funciones ejecutivas en tareas que requieren de inhibición, control de impulsividad, planificación, estructuración de normas y velocidad psicomotriz.

García, García y Secades (2011) y Hester y Garavan (2004) obtuvieron un perfil neuropsicológico que concuerda con los demás autores en torno a las disfunciones cognitivas, motivacionales, emocionales y conductuales que modifican el funcionamiento normal del sujeto en su vida diaria, por las alteraciones en los procesos cognitivos de atención, concentración, integración, procesamiento de la información, ejecución de planes de acción, flexibilidad mental, toma de decisiones e inhibición de respuestas.

Otros autores han estudiado las alteraciones cognitivas con instrumentos neuropsicológicos más sensibles y sencillos, como es el caso del Montreal Cognitive Assesment (MoCA), encontrando que los 79 consumidores participantes en el estudio tuvieron un rendimiento inferior al del grupo control y similar al de pacientes con demencias en tareas que involucran funciones cognitivas como memoria, atención y funciones ejecutivas; sin embargo, los resultados indican un rendimiento significativamente inferior para el caso de 
fluidez verbal en el grupo de adictos (Rojo, Pedrero, Ruiz, Llanero \& Puerta, 2013).

El estudio desarrollado por Woicik et al. (2009) da a conocer mayores disfunciones en las funciones cognitivas de atención y memoria verbal producto de las alteraciones en el cíngulo anterior, la corteza prefrontal anterior izquierda y el hipocampo; regiones que también se encuentran relacionadas con los periodos de abstinencia, las conductas de búsqueda, las recaídas y las dificultades en el aprendizaje.

En cuanto al uso recreacional del principio activo del bazuco, el estudio realizado por Vonmoos et al. (2013) expone la presencia de mayores dificultades de rendimiento en los dominos de atención, memoria de trabajo, memoria declarativa y funciones ejecutivas; sin embargo, las alteraciones más marcadas se encontraron en atención y memoria de trabajo en un grupo de adolescentes.

La revisión sistemática de la literatura llevada a cabo por Czermainski (2017) resalta que se presenta un bajo control inhibitorio, acompañado de altos niveles de impulsividad que ocasionan falencias en el proceso cognitivo y automonitoreo, llevando a que se instaure un factor de riesgo para el consumo de la sustancia y posterior recaída durante el proceso de rehabilitación.

Finalmente, y retomando a Rosseli et al. (1994), es viable plantear que los consumidores de bazuco presentan bajas puntuaciones en instrumentos neuropsicológicos de acuerdo al tiempo de consumo en pruebas de memoria y abstracción, aspectos reflejados en el perfil neuropsicológico y social que obtuvieron caracterizado por: (a) Retardo en el desarrollo psicomotriz, asociado con problemas de hiperactividad, desviaciones de conducta, defectos atencionales, problemas de relaciones interpersonales y, en general, una historia inapropiada de desarrollo; (b) presencia de posibles rasgos de depresión y ansiedad; (c) historia familiar positiva de drogadicción, (d) dificultades en todo el sistema de relaciones interpersonales y sociales; (e) existencia aumentada de patologías psiquiátricas familiares; y $(f)$ frecuencia aumentada de conductas desviadas (p. 103).

A continuación se expondrá la neurotoxicidad que se produce por algunos de los componentes del bazuco, entre los que se encuentran: el permanganato de potasio, el plomo y los solventes (benceno, xileno y tolueno); dicha neurotoxicidad es la responsable de las diferentes alteraciones estructurales y funcionales que se producen tanto en el sistema nervioso central como en el sistema nervioso periférico (Martínez \& Mejía, 2009). A partir de ello, se busca profundizar en las disfunciones cognitivas que se generan en los sujetos dado su consumo crónico, haciendo especial énfasis en que la intensidad o gravedad de dichas alteraciones están mediadas por la concentración, la frecuencia de exposición a la sustancia y el tiempo de consumo.

\section{Permanganato de Potasio}

Según lo expuesto por Uribe (2009), dicha sustancia influye directamente en los procesos cognitivos de atención, 
memoria, juicio, abstracción y raciocinio. Paralelamente, Kalwa y Habrat (2015) describen que esta sustancia produce múltiples desórdenes neurológicos y del movimiento, así como alteraciones en las funciones ejecutivas, memoria de trabajo, procesos atencionales, déficits psicomotores y desórdenes de la emisión o articulación del lenguaje como la disartria.

\section{Plomo}

Esta sustancia tiene la capacidad de alterar el funcionamiento cognitivo a través de dos mecanismos: el primero de ellos corresponde a los cambios estructurales en las neuronas tanto del sistema nervioso central como del sistema nervioso periférico; y el segundo, relacionado con el cambio funcional en los sistemas de neurotransmisión colinérgico y adrenérgico al atravesar la barrera hematoencefálica, de ahí que los consumidores presentan bajo desempeño en la ejecución de tareas que buscan evaluar la inteligencia, encontrando alta distractibilidad, mayores tiempos de reacción, déficit en tareas de vigilancia, bajo aprendizaje, bajo rendimiento en tareas de escritura, lectura, matemática y reconocimiento de palabras, así como decrementos en la memoria a largo plazo, la abstracción verbal, habilidades visoespaciales y la rapidez psicomotora (Martínez \& Mejía, 2009).

En concentraciones bajas, se han encontrado alteraciones emocionales relacionadas con la presencia de ansiedad, tensión, depresión, hostilidad, alucinaciones, agitación junto con bajo rendimiento mnémico, alteraciones psicomotoras, dificultades en habilidades visoespaciales y la formación de conceptos verbales (Martínez \& Mejía, 2009).

\section{Solventes (xileno, benceno y tolueno)}

El benceno, el tolueno y el xileno tienen la capacidad de producir cambios a nivel cerebral que repercuten directamente en funciones cognitivas como la memoria, el lenguaje (comprensión), las funciones ejecutivas (habilidades visoespaciales), la atención (concentración) y en aspectos emocionales relacionados con los rasgos ansiosos en la personalidad (Benito, Barrios \& Girón, 2005). De igual forma, estudios realizados en adolescentes que utilizan dichos solventes reportaron decremento en funciones como la atención, la memoria declarativa, la habilidad motora, la velocidad psicomotora, las habilidades visuales y espaciales, letargo y ataxia (Lara et al., 2003). Finalmente, los solventes producen síntomas como sensación de borrachera, ataxia, somnolencia, cansancio físico y mental (Martínez \& Mejía, 2009).

Por otra parte, Maizlish y Feo (1994) plantean que la exposición al tolueno ocasiona un deterioro en la memoria visual, atención, aprendizaje y destreza manual. Dichos hallazgos concuerdan con los encontrados por Sánchez, Prado, León y González (2014) en su investigación longitudinal de cinco años, en los que se estudió a trabajadores expuestos al tolueno y en los que se concluye que este elemento se asocia con deficiencias en la atención, el aumento en los tiempos de reacción, la distractibilidad y el detrimento de la función motora. Boey, Foo y Jeyaratnam (1997) evidenciaron dificultades 
en el escaneo visual, disminución en la velocidad perceptual y motora, además de decremento en la memoria a corto plazo y en la destreza manual. En cuanto al xileno, Burbacher (1993) menciona que los principales efectos neurotóxicos de esta sustancia se relaciona con el aumento en los tiempos de reacción, la disminución en la destreza manual, la pérdida del equilibrio y la disminución del spam de memoria.

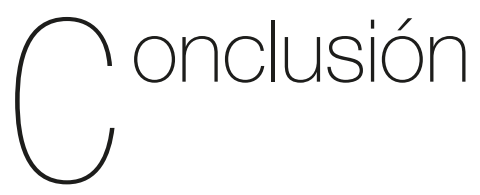

De acuerdo a la información presentada en esta revisión, es posible afirmar que el consumo de bazuco puede conducir a diversas deficiencias cognitivas en los dominios de atención, memoria y funciones ejecutivas; considerando la influencia del tiempo, la frecuencia de consumo y la exposición a solventes como el benceno, el xileno, el tolueno y el permanganato de potasio, al ser moduladores de la severidad de las alteraciones. Sin embargo, éstas no son las únicas dificultades que se presentan, puesto que pueden darse otras en la concentración, la disminución de la velocidad psicomotriz, detrimento de las habilidades visoespaciales, impulsividad, perseveraciones, agresividad, y sintomatología ansiosa y depresiva. Aspectos que, en su conjunto, dificultan la adaptación a la cotidianidad y representan un factor de riesgo para la integridad personal y de terceros, lo que incrementa las problemáticas de salud pública e impacta de manera negativa en el área de la salud mental.
No obstante, se debe tener en cuenta que existen controversias y contradicciones en torno a las alteraciones como parte de los procesos de evaluación ejecutados por los investigadores al utilizar gran variedad de instrumentos de evaluación para inferirir el rendimiento cognitivo.

Las dificultades expuestas poseen un correlato biológico que posibilita una mayor comprensión de los mecanismos que intervienen en el desarrollo de problemáticas a nivel psicológico y social, dadas por los cambios estructurales y funcionales en la sustancias neuroquímicas que intervienen en el sistema de recompensa y cuyo rol es definitivo en la instauración de la adicción.

Otro aspecto a destacar de los hallazgos es la predominancia de estudios transversales que dificultan la comprensión de las alteraciones neurocognitivas a largo plazo, al no existir claridad en los dominios implicados y la posible restauración de funciones superiores en diferentes tiempos de abstinencia. A esto se suma el policonsumo de los participantes incluidos en los estudios, ya que las diferentes sustancias que acompañan el consumo de bazuco intervienen en el funcionamiento del sistema nervioso. Incluso, la presencia de comorbilidades psiquiátricas puede generar cambios en el rendimiento cognitivo de los participantes.

Para finalizar, es menester destacar la importancia de futuras investigaciones orientadas a individuos adictos al bazuco con el fin de profundizar en los efectos cognitivos y neurales de la sustancia, ampliando así el respaldo científico que sustentará el diseño de tratamientos eficaces en el manejo de la adicción con base en las repercusiones neuropsicológicas. 


\section{Referencias}

Ambrosio, E. (2008). Efectos de la cocaína en el ser humano. Trastornos Adictivos, 10(3), 151-165.

Areal, L. B., Rodrigues, L. C. M., Andrich, F., Moraes, L. S., Cicilini, M. A., Mendonça, J. B., ... Pires, R. G. W. (2015). Behavioural, biochemical and molecular changes induced by chronic crack cocaine inhalation in mice: The role of dopaminergic and endocannabinoid systems in the prefrontal cortex. Behavioural Brain Research, 290, 8-16. doi:10.1016/j.bbr.2015.04.036

Batista, E. K., Klauss, J., Fregni, F., Nitsche, M. A., \& Nakamura-Palacios, E. M. (2015). A Randomized Placebo-Controlled Trial of Targeted Prefrontal Cortex Modulation with Bilateral tDCS in Patients with CrackCocaine Dependence. International Journal of Neuropsychopharmacology, 18(12), pyv066. doi:10.1093/ijnp/pyv066

Benito, M., Barrios, L. \& Girón, V. (2005). Estudio de alteraciones neuropsicológicas en sujetos expuestos y no expuestos a solventes orgánicos en fábricas de pintura de la ciudad de Bogotá. Cuadernos Hispanoamericanos de Psicología, 5(1), 77-91.

Benowitz, N. (2004). Cocaine. En Olson, K. (Ed.), Poisoning \& drug overdose (pp. 171 173). California, EU: McGraw-Hill.

Boey, K., Foo, S. \& Jeyaratman, J. (1997). Effects of occupational exposure to toluene: a neuropsychological study on workers in Singapore. Annals of the Academy of Medicine, Singapore, 26(2), 184-187.

Bolla, K., Ernst, M., Kiehl, K., Mouratidis, N., Eldreth, D., Contoreggi, C.,... London, E. (2004). Prefrontal cortical dysfunction in abstinent cocaine abusers. Journal of Neuropsychiatry \& Clinical Neurosciences, 16(4), 456-464.

Bolla, K., Rothman, R. \& Cadet, J. (1999). Dose-related neurobehavioural effects of chronic cocaine use. Journal of Neuropsychiatry \& Clininical Neurosciences, 11(3), 361369.

Burbacher, T. (1993). Neurotoxic effects of gasoline and gasoline constituents. Enviromental Health Perspectives Supplements, 101(6), 133-141.

Callado, L. (2001). Neurobiología de la drogadicción II. cocaína y drogas de diseño. Osasunaz (4), 197-210.

Casale, J. \& Klein, R. (1993). Illicit production of cocaine. Forensic Science Review, 5(2), 95-107.

Castaño, G. (2000). Cocaínas fumables en Latinoamérica. Adicciones, 12(4), 541-550.

Conti, C. L., \& Nakamura-Palacios, E. M. (2014). Bilateral transcranial direct current stimulation over dorsolateral prefrontal cortex changes the drug-cued reactivity in the anterior cingulate cortex of crackcocaine addicts. Brain Stimulation, 7(1), 130-132. doi:10.1016/j.brs.2013.09.007

Cooper, J. (1982). The biochemical basis of neuropharmacology. New York, EU: Oxford Press. 
Córdoba, D. \& Toledo, D. (2001). Cocaína y base de cocaína bazuco. En D. Córdoba (Ed.), Toxicología (pp. 445-449). Bogotá D.C, Colombia: El Manual Moderno.

Corominas, M., Roncero, C., Bruguera, E., \& Casas, M. (2007). Sistema dopaminérgico y adicciones. Revista de Neurología, 44(1), 23-31.

Czermainski, F. R., Willhelm, A. R., Santos, Á. Z., Pachado, M. P., \& de Almeida, R. M. M. (2017). Assessment of inhibitory control in crack and/or cocaine users: a systematic review. Trends in Psychiatry and Psychotherapy, 39(3), 216-225. doi:10.1590/2237-6089-2016-0043

Damin, C. \& Grau, G. (2015). Cocaína. Acta Bioquímica Clínica Latinoaméricana, 49(1), 127-134.

Delgado, H. (2011). Alteraciones del flujo sanguíneo cerebral en consumidores activos de pasta base y clorhidrato de cocaína (Tesis de Grado). Universidad de la República Uruguay, Uruguay.

Dreisbach, A., Robertson, W. \& William, O. (1999). Manual de toxicología clínica: prevención, diagnóstico y tratamiento. México DF: Editorial Manual Moderno.

Echeverry, J., Moya, M., Zapata, P., \& Martínez, J. (2004). Actividad motora, ánimo y sueño durante los primeros quince días de abstinencia de base de cocaína. Revista Médica de Risaralda, 10(1), 13-20.

Fein, G., Di Sclafani, V., \& Meyerhoff, D. J. (2002). Prefrontal cortical volume reduction associated with frontal cortex function deficit in 6-week abstinent crack-cocaine dependent men. Drug and Alcohol
Dependence, 68(1), 87-93. doi:10.1016/ s0376-8716(02)00110-2

Fernández, E. (2006). Neurobiología de la adicción a psicoestimulantes. Revista de Neurología, 43(3), 147-154.

Ferrando, R., Bochino, S., Barrachina, A., Ferro, A., Rodríguez, J., Silveira, A., Ventura, R.,... Lago, G. (2009). Alteraciones de la perfusión cerebral en consumidores activos de pasta base de cocaína. Revista de Psiquiatría del Uruguay, 73(1), 51-62.

Gainza, I., Nogué, S., Martínez, C., Hoffman, R., Burillo, G., Dueñas, A.,... Pinillos, M. (2003). Intoxicación por drogas. ANALES del Sistema Sanitario de Navarra, 26(1), 99-128.

García, G., García, O. \& Secades, R. (2011). Neuropsicología y adicción a drogas. Papeles del Psicólogo, 32(2), 159-165.

García, R. C. T., Dati, L. M. M., Fukuda, S., Torres, L. H. L., Moura, S., de Carvalho, N. D., ...Marcourakis, T. (2012). Neurotoxicity of Anhydroecgonine Methyl Ester, a Crack Cocaine Pyrolysis Product. Toxicological Sciences, 128(1), 223-234. doi:10.1093/ toxsci/kfs140

Garro, K. (2011). Cocaína: actualización médico legal. Medicina Legal de Costa Rica, 28(2), 57-62.

Garza-Villarreal, E. A., Chakravarty, M., Hansen, B., Eskildsen, S. F., Devenyi, G. A., CastilloPadilla, D., ... Gonzalez-Olvera, J. J. (2017). The effect of crack cocaine addiction and age on the microstructure and morphology of the human striatum and thalamus using shape analysis and fast diffusion kurtosis imaging. Translational Psychiatry, 7(5), e1122. doi:10.1038/tp.2017.92 
Gobierno Nacional de la República de Colombia. (2014). Estudio Naciona de Sustancias Psicoactivas en Colombia 2013. Recuperado de https://www.unodc. org/documents/colombia/2014/Julio/ Estudio_de_Consumo_UNODC.pdf

Hester, R. \& Garavan, H. (2004). Executive dysfunction in cocaine addiction: evidence for discordant frontal, cingulate and cerebellar activity. The Journal of Neuroscience, 24(49), 11017-11022. doi:10.1523/JNEUROSCI.3321-04.2004

Kalwa, A. \& Habrat, B. (2015). Cognitive dysfunctions caused by excessive exposure to manganese compounds. Cognitive disturbances in intravenous users of ephedrone (methcathinone) with manganese compounds. Psychiatria Polska, 49(2), 305-314. http://dx.doi. org/10.12740/PP/28048

Lara, M., Galindo, G., Romero, M., Salvador, J. \& Domínguez, M. (2003). La figura compleja de rey en adolescentes que consumen disolventes inhalables. Salud Mental, 26(6), 17-26.

Lima, L. (2007). Neurobiología de la adicción a drogas. Archivos Venezolanos de Psiquiatría y Neurología, 53(109), 14-24.

Lipaus, I. F. S., Gomes, E. F., Martins, C. W., e Silva, C. M., Pires, R. G. W., Malgarin, F., ... de Melo Rodrigues, L. C. (2018). Impairment of spatial working memory and oxidative stress induced by repeated crack cocaine inhalation in rats. Behavioral Brain, 359, 910-917. doi:10.1016/j.bbr.2018.06.020

Lizasoain, I., Moro, M. \& Lorenzo, P. (2002). Cocaína: aspectos farmacológicos. Adicciones, 14(1), 57-64.
López, A. \& Becoña, E. (2006). El craving en personas dependientes de la cocaína. Anales de Psicología, 22(2), 205-211.

Madoz, A. \& Ochoa, E. (2012). Alteraciones de funciones cognitivas y ejecutivas en pacientes dependientes de cocaína: estudio de casos y controles. Revista de Neurología, 54(4), 199-208.

Maizlish, N. \& Feo, O. (1994). Alteraciones neuropsicológicas en trabajadores expuestos a neurotóxicos. Salud de los Trabajadores, 2(1), 5-34.

Manschreck, T., Laughery, J., Weissteil, C., Allen, D., Humblestone, B., Neville, M.,... Mitra, N. (1988). Characteristics of freebase cocaine psychosis. The Yale Journal of Biology and Medicine, 61(2), 115-122.

Martínez, A. \& Mejía, M. (2009). Alteraciones neuropsicológicas en síndromes neurotóxicos. En G. Pérez \& M. Uribe (Eds.), Neurotoxicología (pp. 333-354). Bogotá D.C, Colombia: Asociación Colombiana de Neurología.

Martínez, J., Aguilar, E., \& Rosales, V. (2006). Bases fisiopatológicas del daño renal causado por la cocaína. Medicina Interna de México, 22(5), 403-410.

Meikle, M., Urbanavicius, J., Prunell, G., Umpiérrez, E., Carriquiri, A., \& Scorza, N. (2009). Primer estudio preclínico de la adicción a pasta base de cocaína en el sistema nervioso central. Revista de Psiquiatría de Uruguay, 73(1), 25-36.

Méndez, P. \& Murillo, H. (2013). El Paco: un proyecto de muerte. casa faro: un proyecto de vida de diagnóstico y derivación "casa faro" Ajayu, 11(1), 34-64. 
Motta, G. (1984). Una farmacodependencia epidemica: bazuco. Revista Colombiana de Psiquiatría, 13(1), 20-27.

National Institute of Drug Abuse. (2010). Cocaína: abuso y adicción. Recuperado de https://d14rmgtrwzf5a.cloudfront.net/ sites/default/files/rrcocaina.pdf

O'Brien, C. (2001). Drug addiction and drug abuse. En J. Shanahan \& C. Naglieri, (Eds.), Goodman and Gilman's: The pharmacological basis of therapeutics (pp. 621-667). Nueva York, EE.UU: McGraw Hill.

Observatorio Colombiano de Drogas (2016). Reporte de Drogas en Colombia 2016. Recuperado de http://www.odc.gov. co/Portals/1/publicaciones/pdf/odclibro-blanco/ODC0100322016_reporte_ drogas_colombia_2016.pdf

Observatorio Uruguayo de Drogas. (2014). Pasta Base Cocaína en Uruguay. Recuperado de https://www. academia.edu/9951331/Pasta_base_ de_coca\%C3\%ADna_en_Uruguay_ compilaci\%C3\%B3n_?auto=download

Oficina de las Naciones Unidas Contra la Droga y el Delito. (2013). Pasta básica de cocaína cuatro décadas de historia, actualidad y desafíos. Recuperado de https://www.unodc.org/documents/ peruandecuador/Publicaciones/ Publicaciones2013/LIBRO_PBC.pdf

Organización de los Estados Americanos \& Comisión Interamericana para el Control del Abuso de Drogas. (2014). Consumo de pasta base de cocaína en américa del sur: revisión de los aspectos epidemiológicos y médico-toxicológico. Recuperado de http://www.cicad.oas.org/oid/pubs/pbc. pdf

Organización de los Estados Americanos, Comisión Interamericana para el Control del Abuso de Drogas, Organización Panamericana de la Salud \& Organización Mundial de la Salud. (2005). Neurociencia del consumo y dependencia de sustancias psicoactivas. Recuperado de http://www. who.int/substance_abuse/publications/ neuroscience_spanish.pdf

Ortega, A. (2003). ¿Estaba intoxicado por cocaína este individuo? (y ii): estimaciones basadas en los efectos de la droga y en las concentraciones de benzoilecgonina. Cuadernos de Medicina Forense, 9(33), 13-24.

Pascale, A., Negrin, A. \& Laborde, A. (2010). Pasta Base de Cocaína: experiencia del centro de información de asesoramiento toxicológico. Observatorio Uruguayo de Drogas, 22(3), 45-54.

Pereiro, C. (2011). Patalogía Dual: trastornos de ansiedad. En J. Bobes, M. Casas, \& M. Gutiérrez. (Eds.), Manual de trastornos adictivos (pp. 159-165). España: Enfoque Editorial, S.C.

Periañez, J. \& Ríos, M. (2012). Neuroanatomía y neuroimagen de la corteza prefrontal. En J. Tirapu., A. García., M. Ríos \& A. Ardila. (Eds.) Neuropsicología de la corteza prefrontal y de las funciones ejecutivas (pp. 55-86). España: Viguera.

Rojo, G., Pedrero, E., Ruiz, J., Llanero, M., \& Puerta, C. (2013). Cribado neurocognitivo en adictos a sustancias: la evaluación cognitiva de montreal. Revista de Neurología, 56(3), 129-136. 
Roncero, J., Ramos, J., Collazos, F., \& Casas, M. (2001). Complicaciones psicóticas del consumo de cocaína. Adicciones, 13(2), 179-189.

Rosselli, M., Rico, S., Sandoval, V., Sierra, Y., \& Uribe, A. (1994). Efectos cognoscitivos del uso del bazuco y la cocaína. Suma Psicológica, 1(1), 88-103.

Sabogal, J. \& Urrego, J. (2012). Composición química de muestras de bazuco incautado en colombia primer semestre de 2010. Revista de Salud pública 14(6), 1014-1025.

Samoel Rodrigues, V., Ribeiro, L., Arruda Rodrigues, L., Quarti Irigaray, T., De Almeida Ribeiro, F., \& Da Silva Oliveira, M. (2017). Correlations Between Cognitive Performance and Readiness to Change in Cocaine/Crack users. Universitas Psychologica, 16(2), 5-14. doi:10.11144/ javeriana.upsy16-2.ccpr

Sánchez, B., Prado, L., León, S., \& González, R. (2014). Trabajadores expuestos a solventes y daños a la salud: una revisión sistemática. Revista Colombiana de Salud Ocupacional, 4(4), 25-29.

Sánchez, L. (2006). El bazuco: ¿Una dramática salida de la drogadicción? En L. Sánchez (Ed.), Mito y realidad de la drogadicción. Bogotá: San Pablo.

Sánchez, N. \& Belistri, U. (2007). Problemas planteados por el consumo de PBC en la psiquiatría forense. Revista de Psiquiatría del Uruguay, 71(1), 9-19.

Schuch-Goi, S. B., Goi, P. D., Bermudez, M., Fara, L. S., Kessler, F. P., Pechansky, F., ... von Diemen, L. (2017). Accumbens volumesarereducedamong crack-cocaine users. Neuroscience Letters, 645, 86-89. doi:10.1016/j. neulet.2017.02.073

Scoppetta, O. (2010). Consumo de drogas en Colombia: Características y tendencias. Bogotá: Editorial Guadalupe.

Smith, J. \& Dworkin, S. (2000). Brain structures and drugs. Encyclopedia of drugs, alcohol and addictive behavior. New York, EU: Macmillan Reference USA. Recuperado de http://www.encyclopedia. com/doc/1G2-3403100089.html

Souza, M. \& Cruz, L. (2008). Nosografía y manejo de la patología asociada al consumo de cocaína. Revista Mexicana de Neurociencia, 9(2), 141-149.

Téllez, J. \& Cote, M. (2005). Efectos toxicológicos y neuropsiquiátricos producidos por consumo de cocaína. Revista de la Facultad de Medicina de la Universidad Nacional de Colombia, 53(1), 10-26.

Uribe, M. (2009). Neurotoxicidad por sustancias de abuso. En G. Pérez \& M. Uribe. (Eds.), Neurotoxicología (pp. 5573). Bogotá D.C, Colombia: Asociación Colombiana de Neurología.

Urigüen, L. \& Callado, L. (2010). Cocaína y cerebro. Trastornos Adictivos, 12(4), 129-134.

Volkow, N., Wang, G., Fowler, J., Tomasi, D. \& Telang, F. (2010). Addiction: beyond dopamine reward circuitry. Proceedings of the National Academy of Sciences, 108(37), 15037-15042.

Vonmoos, M., Hulka, L., Preller, K., Jenni, D., Baumgartner, M., Stohler, R.,..., Quednow, 
B. (2013). Cognitive dysfunctions in Woicik, P., Moelle, S., Klein, N., Maloney, T., recreational and dependent cocaine Lukasik, T., Yeliosof, O.,..., Goldstein, R. users: role of attention-deficit hyperactivity (2009). The neuropsychology of cocaine disorder, craving and early age at onset. addiction: recent cocaine use masks The British Journal of Psychiatry, 203(1), impairment. Neuropsychopharmachology, 35-43. doi: 10.1192/bjp.bp.112.118091 34(5), 1112-1122. doi: 10.1038/npp.2008.60 